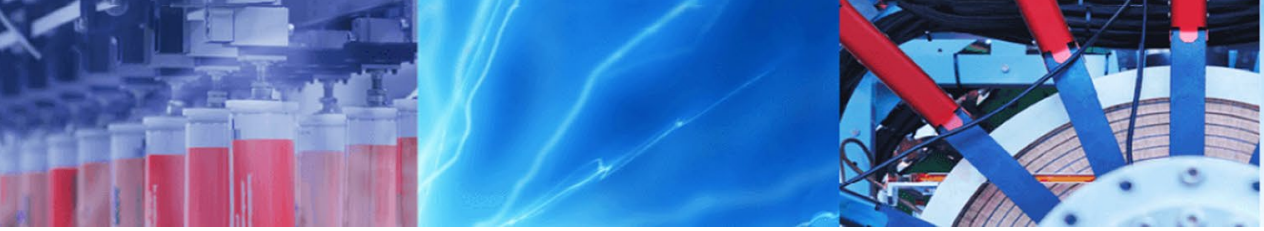

Research Article

\title{
Parametric analysis and soft-computing prediction of sweet potatoes (Ipomoea batatas $L$ ) starch drying using machine learning techniques
}

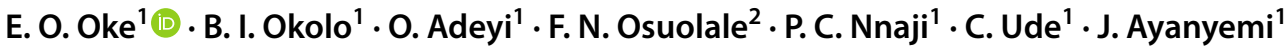

Received: 9 December 2019 / Accepted: 19 August 2020 / Published online: 26 August 2020

(c) Springer Nature Switzerland AG 2020

\begin{abstract}
This study is based on parametric selection and prediction of sweet potatoes starch drying using Regression Tree (RT), Support Vector Machine (SVM) and Neuro-Fuzzy (NF) soft-computing techniques. The drying experiments are conducted at Drying Temperature (DT): $40-60{ }^{\circ} \mathrm{C}$, Drying Time (DTi): 0-780 min, Ambient Temperature (AT): $27.2-30^{\circ} \mathrm{C}$ and Relative Humidity (RH): $70-80 \%$. Exhaustive search model is used to determine the most and least relevant drying parameters. NF, RT and SVM programming codes are developed in Matlab 9.2 (2017a) with four, three and two-input variable combination NFs (4-1NF, 3-1NF and 2-1NF), RTs (4-1 RT, 3-1 RT and 2-1 RT) and SVMs (4-1SVM, 3-1SVM and 2-1SVM) for the prediction of the starch drying. Exhaustive NF parametric analysis results show that DT-DTi-AT and DT-DTi are the most influential combined variables for three and two variables combinations respectively. DTi and RH are also the most and least influential parameters, respectively. The 3-1NF with neighbourhood radius 0.7 gave the uppermost correlation coefficient $\left(R^{2}\right)$ 0.999; and the lowermost root mean square error as well as mean square error 0.0025 and 0.00000625 respectively. The results obtained show that exhaustive search and 3-1NF models are suitable for the prediction of sweet potatoes starch drying.
\end{abstract}

Keywords Exhaustive search $\cdot$ Sweet potatoes $\cdot$ Starch drying $\cdot$ Neuro-fuzzy

\section{Introduction}

Sweet potato (Ipomoea batatas $L$.) is a significant tuber crops in the world. China is globally ranked as the main producer of sweet potato; while Nigeria is categorised as the second producer in Africa and third in the world [1]. Sweet potato has been a high starch-yielding tuber crop with the advantage of harvesting twice in a year as cassava crop [2]. A high amount of sweet potato tuber harvested during the peak season is significantly deteriorating due to poor storability and low marketability. Recently, the government has emphasised the exportation of industrial product derived from agricultural produce in Nigeria. Thus, there is a need to transform the unprocessed sweet potato into a more usable and refined form, such as dried sweet potato starch, for industrial applications.

Starch is one of the essential ingredients in pharmaceutical, food, cosmetic and petroleum industries. Starch pastes are used as thickeners and other additive ingredients in food systems and pharmaceutical sectors [3-5]. It is a white and tasteless complex polymer, which is plentifully obtained in large quantities in roots and tubers such as cassava, yam, and potato [5-7]. Previous researches show that the starch from potato has a high hot paste viscosity, unlike cereal starches, which makes it preferable for the manufacture of adhesives $[8,9]$. Starch is conventionally prepared from the source material through the extraction and drying processes. Among the unit operations involved in the production of dried starch, the drying operation

E. O. Oke, solaemmanuel1@gmail.com | ${ }^{1}$ Chemical Engineering Department, Michael Okpara University of Agriculture, Abia State, Umudike, Nigeria. ${ }^{2}$ Chemical Engineering Department, Ladoke Akintola University of Technology, Ogbomoso, Nigeria. 
is fundamentally ultimate. It extends material shelf life, reducing its deteriorating potential and also decreases product mass-volume ratio and, consequently minimizes the transportation cost.

Drying is a complex solid-liquid separation process which involves moisture removal from the solid matrix of agricultural produce. Moisture removal from food material requires thermal energy to create vapour pressure gradient between the material and ambient drying environment. Amount of moisture removal from solid matrix occurs due to diffusion of water or water vapour through the solid structure; consequently, transient simultaneous heat and mass transfer occur at the food material surface $[10,11]$. The migration of vapour from the food surface to the drying air and the transference of heat from the air to the product surface depend on the existing vapour pressure and temperature gradients, respectively. Drying operation has been used to preserve various agricultural products [12-14] and it was reported that reasonable percentages of moisture were removed from the materials.

The amount of moisture removed from food material during dehydration is affected by various parameters such as air drying temperature, air velocity, initial moisture content, sample thickness and drying chamber relative humidity $[15,16]$. Parametric study of the process is necessary so as to have better knowledge of process variable importance and relevancy [17]. Reported that models are often used for the selection of the relevant variables involved in the process prediction and optimization of the product. Mathematical model describing drying process serves as a monitoring tool to achieve better control of the unit operation in order to protect the quality of the product [18]. Prediction of moisture removal during food drying has been achieved through empirical and softcomputing techniques $[19,20]$.

Numerous soft-computational techniques such as neural-network, fuzzy logic, genetic algorithm, neurofuzzy (genfis1, genfis2 and genfis3), support vector machine, decision tree, multi-linear regression have been applied for solving complex problems in various engineering researches. The ability of the techniques to establish the relationship between input/output variable without prior information, unlike physics models, about the process makes them useful in recent time. On contrary, theoretical models are cumbersome and difficult to solve due to the fact that most of the problems resulting to initial boundary-value equations. Countless process predictions have been achieved through soft-computational methods [21-23]. Forecasted performance of an orange juice spray drier using ANNs. Also, [24] compared ANN approach with response surface methodology (RSM) technique for modelling the quality parameters of spray-dried pomegranate juice [25]. Designed a fuzzy logic controller for grain drying in order to obtain the grain output moisture content near to the reference point in spite of disturbance [26]. Used an ANN model to determine drying behaviour of apples in the freezedrying process while [27] developed a fuzzy logic-based control system for a solar dryer.

Various researches have been conducted on sweet potatoes starch extraction [28, 29], drying [30,31] and its applications [32, 33]. However, based on our scientific hunt, information about robust soft-computing prediction and parametric analysis of starch drying is rarely found in the pool of literature. Soft-computing methods such as regression tree, adaptive neuro-fuzzy inference system and support vector machine can adequately diagnose and identify the interactions between process variables of complex and non-linear processes. Thus, this paper is structured and sectioned as follows: (1) starch extraction from sweet potatoes tuber and drying experimental study of sweet potatoes starch (2) grid-partitioning neuro-fuzyy exhaustive search drying input parametric analysis for selecting the most and least relevant drying variables ( 3 ) sub-clustering neuro-fuzzy adaptive neuro-fuzzy, regression tree and support vector Matlab programming code development for the prediction of sweet potatoes starch drying.

\section{Material and method}

\subsection{Sample collection}

The sweet potato tubers used in this study was obtained from National Root Crops Research Institute, Umudike, Nigeria. The species of the sweet potato was called Orange Fleshed sweet potato. The tubers were washed in order to get rid of soil and dirt stick to the sample.

\subsection{Starch extraction and chemical analyses}

Starches were isolated according to the method of [5]. $25 \mathrm{~kg}$ tubers were skinned, thoroughly clean with water and crushed. The peeled tubers were crushed in a locally fabricated grater. The crushed pulp was mixed with clean water and the fibre separated by passing through a $75 \mathrm{~mm}$ sieve. The mixture (starch milk) was settled for ten hours and the supernatant was decanted. The starch was washed repeatedly with fresh water. Then, the starch was moulded into definite geometry using a rectangular mould. The initial moisture content of the starch was evaluated in a drying oven at $105^{\circ} \mathrm{C}$ for $48 \mathrm{~h}$ by $\mathrm{AOAC}$ method [34]. 


\subsection{Drying experimentation}

Convective laboratory tray dryer (Heratherm Oven CP 210,997), as diagrammatically shown in Fig. 1, was used for drying the starch. The dimensions of the drying chamber were $360 \times 620 \times 460 \mathrm{~mm}$ and consist of 3 trays of $327 \times 405 \mathrm{~mm}$. Heating elements were fixed at the inner back of the dryer. The degree of temperature of the drying chamber was meticulously monitored with a temperaturebased controller imbedded into the tray dryer. Air was sucked rom ambient environment and distributed into the dryer with a fan at the back of the dryer. The air velocity inside the chamber was measured as $1.5 \mathrm{~m} / \mathrm{s}$ with a digital anemometer (Model PM6252A) with accuracy of \pm 0.1 . The dryer was allowed to preheat to the desired temperature, and the starch was spread onto the trays in single layer for a specified time. The ambient, inlet and outlet temperature and relative humidity of the dry air were measured with a squared multi-thermometer (Model TA298) with an accuracy of $\pm 0.1^{\circ} \mathrm{C}$. The components of schematic dryer diagram in Fig. 1 are as follows: (1) Outdoor door (2) Door latch cut out (3) Door latch and handle (4) Door joint subordinate (5) Levelling base (6) Nameplate (7) Air battle top piece (8) Support rail for wire mesh shelf (9) Shelf pivot (10) Fan cover (11) Door hook catch (12) Air baffle (13) Door seal (14) Stacking pad (15) Spring for air baffle (16) Temperature sensor (17) Exhaust air tube. The final moisture content of the dried starch was evaluated by AOAC method [34]:

$\mathrm{MC}=\frac{W_{i}-W_{f}}{W_{f}} \times 100$

where $M C$ is the dry basis $(\mathrm{db})$ moisture content in $\%$; $W \mathrm{i}$ is the weight of sample taken in $\mathrm{g}$ for drying at $105^{\circ} \mathrm{C}$; and $W f$ is the weight sample in $\mathrm{g}$ after drying at $105^{\circ} \mathrm{C}$.

The proximate compositions of dried starch at different temperatures was also determined by [34].

\subsection{Exhaustive search and subclustering Neuro-fuzzy modelling}

\subsubsection{Exhaustive search (ES) method for input selection}

Drying temperature, drying time, ambient temperature and relative humidity are the four input attributes of the starch drying operation considered in this study. The predicted output variable is moisture content ratio. The experimental data set were then split into training data and checking data. The 'exhsrch" function in MATLAB software environment was used to execute the search within the input variables and select the most as well as the least relevant inputs that affect the moisture content ratio. ES builds an neural fuzzy model structure as shown in Fig. 2
Fig. 1 Schematic diagram of the dryer

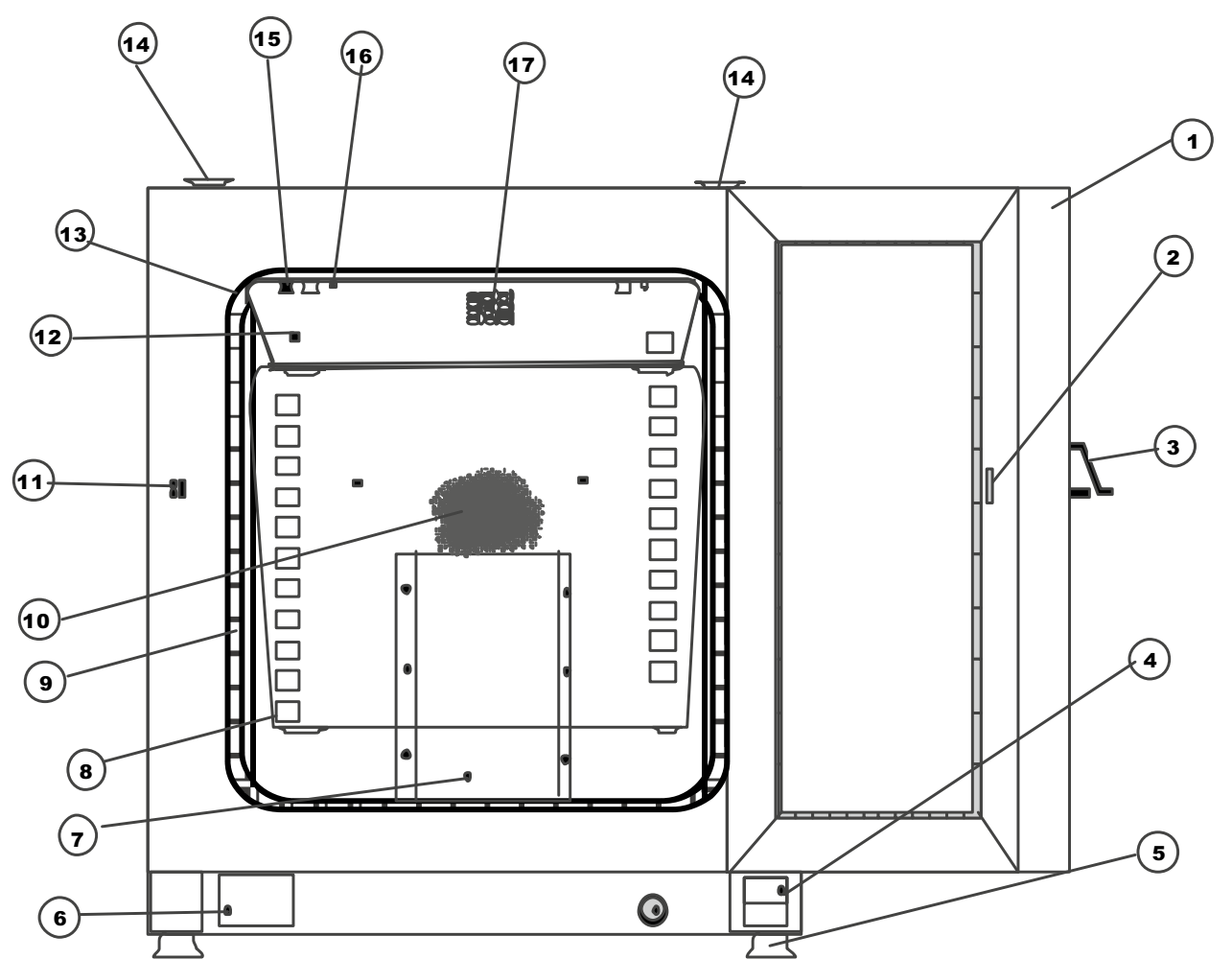

SN Applied Sciences A SPRINGER NATURE journa 
Fig. 2 A basic structure of the ANFIS

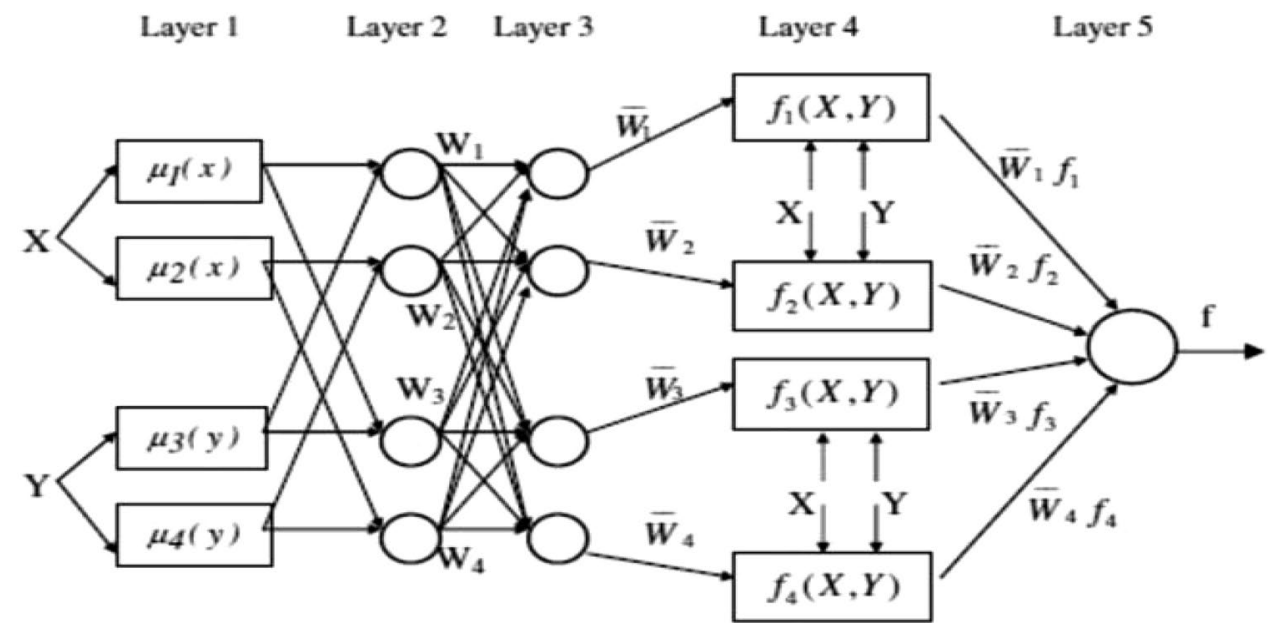

for combination of inputs and trained for one run; then, the performance of the model as reported.

\subsubsection{Fuzzy subtractive clustering and model identification}

The starch input variables (Drying Temperature (DT), Drying Time (DTi), Ambient Temperature (AT) and relative humidity $(\mathrm{RH})$; and output response (moisture ratio) were clustered and converted to fuzzy rule base via subbclustering technique in Matlab software. Clustering technique first estimates numbers of clusters, their centres and radii formed in a data set $[23,35]$. Subclustering algorithm takes each data point from the vector space as cluster centre and also estimate density or potential of the cluster according to Eq. (2).

$C_{i}=\sum_{j}^{m} \exp ^{-\rho\|\cdot\| x_{i}-x_{j}\|\cdot\|}$

where $\rho=4 / r_{a}^{2}$ and $r_{a}>0$ shows the locality length for each cluster midpoint. $C_{1}^{*}=$ strength measure of data point. Equation (3) and (4) show the reduction of strength/potential measure for each cluster centre

$C_{i}=C_{i}-C_{1}^{*} \exp ^{-\sigma\|\cdot\| x_{i}-x_{1}^{*}\|\cdot\|}$.

Let

$\varphi\left(x_{i}\right)=\exp \frac{4\left(\|\cdot\| x_{i}-x_{1}^{*}\|\cdot\|\right)}{r_{b}^{2}}$

Also $\sigma=4 / r_{b}^{2}$ and denotes lenght of neighbourhood where the reduction occurs; $r_{a}$ is called cluster length and $\|$.$\| means the Euclidean distance, Where r_{b}$ is a constant that marks in a considerable reduction in strength measures of the neighbourhood so as to reduce clusterdly cluster centers. Equation (2) was used to generate the density measure of each point and the data point with the highest density measure is allocated the next cluster centre. Then, the clusters' data obtained in Eq. (2) was used for initializing the initial rules number and antecedent membership function for ascertaining the Fuzzy Inference System (FIS) [36]

2.4.2.1 Cluster radius determination and consequent parameters estimation To determine the best cluster radius $\mathrm{r}_{\mathrm{a}}$, subclustering algorithm was implemented with various cluster radii of neighbourhood starting from o to 1 with corresponding fuzzy inerence systems. The prediction performance is estimated as validity index and expressed as:

$e=\frac{1}{N} \sum_{K=1}^{N}(x-\hat{y})$

where $x$ and $\hat{y}$ are the true data and the predicted response respectively.

The consequent variables (ai, bi) are determined from the identification parameter set by least-squares optimization technique. The regressors $(\xi)$ and regress $(y)$ of model data and probability function of the FIS are shown below:

$\beta=\left[\begin{array}{c}\beta_{1}^{T} \\ \beta_{2}^{T} \\ \beta_{N}^{T}\end{array}\right], y=\left[\begin{array}{c}y_{1} \\ y_{2} \\ \vdots \\ \vdots \\ y_{N}\end{array}\right], W_{i}\left[\begin{array}{cccc}\varphi_{i 1} & 0 & \cdots & 0 \\ 0 & \varphi_{i 2} & \cdots & 0 \\ \vdots & \vdots & \vdots & \vdots \\ 0 & 0 & \vdots & \varphi_{i N}\end{array}\right]$

The variables of the logic rule belonging to the ith cluster, ai and $b i$ are collapsed into a single parameter vector $\beta_{i}$ :

$\beta=\left[a_{i}^{T} b_{i}\right]^{T}$ 
$\theta=\left[\beta_{e}^{T} W_{i} \beta_{e}\right]^{-1} \beta_{e}^{T} W_{i} y$

This is the least solution of $y=\beta_{e} \theta+\varepsilon$, where parameters ai and bi are expressed as:

$a_{i}=\left(\theta_{1}, \theta_{2}, \ldots \ldots \ldots \theta_{p}\right)$ and $b_{i}=\theta_{p+1}$

where $\theta i, i=1,2 \ldots n$, can be evaluated by the weighted least-squares technique.

\subsection{Support vector machines (SVMs)}

Support Vector Machine (SVM) is a product of statistical learning concept [37]. SVM structure is similar to ANN architecture with non-linear weight in the first layer and the linear operation in second layer [38]. SVM is used to forecast and predict input-output relationship of a set of system data such as $(x i, y i) n$, Where $x i$ is input variables Drying Temperature (DT), Drying Time (DTi, Ambient Temperature (AT) and relative humidity $(\mathrm{RH})$; while yi shows output variable (moisture ratio). Thus, SVM uses the function in Eq. 6:

$f(x)=\omega . \emptyset(x)+b$.

where $\emptyset($.$) is a complex function by which \mathrm{x}$ is related or plotted into the space, $\mathrm{b}$ and $\omega$ denote a weight vector and a coefficient that are evaluated from the data. $b$ and $\omega$ are evaluated by minimizing the sum of the uncertainty in the first term of function (Eq. 8) and a nonliner term in the second term of function (Eq. 8).

$R=C \sum_{i=1}^{n} L_{g}\left(f\left(x_{i}\right), y_{i}\right) \frac{1}{2}\left\|\omega^{2}\right\|$

$L_{g}\left(f\left(x_{i}\right), y_{i}\right)= \begin{cases}0 & \text { for }|f(x)-y|<\varepsilon \\ |f(x)-y|-\varepsilon & \text { otherwise }\end{cases}$

$f\left(x, \propto, \alpha^{*}\right)=\sum_{i=1}^{N}\left(\alpha_{i}-\alpha^{*}\right) k\left(x_{i}, x\right)+b$.

So, Eq. (9) minimizes Eq. (7) subject to Eq. (11) the following expression where $\alpha_{i} \alpha_{i}^{*}=0, \alpha_{i} \alpha_{i}^{*} \geq 0 i=1, \ldots, N$ and $k\left(x_{i}, x\right)$ shows the inner product in the feature space. Equation 10 is an expression of the kernel function:

$k(x, y)=\sum_{i=1}^{D} \emptyset_{j}(x) \emptyset_{i}(y)$

Different kernels such as linear, polynomial, radial basis function and Sigmoid were used in this investigation in order to determine the best kernel. $\alpha_{i} \alpha_{i}^{*}$ are gotten by maximizing Eq. 14 according to [39-41].

$$
\begin{aligned}
R\left(\alpha^{*} \propto\right)= & -\operatorname{\varepsilon um}_{i-1}^{N}\left(\alpha_{i}^{*}+\alpha_{i}\right)+\sum y_{i}\left(\alpha_{i}^{*}-\alpha_{i}\right) \\
& -\frac{1}{2} \sum_{i j-1}^{N}\left(\alpha_{i}^{*}+\alpha_{i}\right) \times\left(\alpha_{i}^{*}+\alpha_{i}\right) k\left(x_{i}, x_{j}\right) .
\end{aligned}
$$

\subsection{Regression tree model}

Sweet potatoes starch drying data were modelled using Recursive Partitioning (RP) algorithm. RP models are product of Recursive Partitioning algorithm. The algorithm forms a tree by recursively dividing the training data into smaller subdivisions. The RP algorithm accepts as input variables DT, DTi, AT and RH of $n$ data points, $D_{t}=\left\{x_{i}, y_{i}\right\}_{i=1}^{n_{t}}$. Then, the test mode criteria termination was set and implemented; and test node $t$ generated. These subdivision data entail the fragmented test $s^{*}$ in the node $t, D_{t_{L}}=\left\{\boldsymbol{x}_{i}, y_{i} \in D_{t}: \boldsymbol{x}_{i} \rightarrow s^{*}\right\}$, and the remaining cases, $D_{t_{R}}=\left\{x_{i}, y_{i} \in D_{t}: x_{i} \rightarrow s^{*}\right\}$. Then, the greedy hill-climbing algorithm was used at each node and the best split test is considered according to some local criterion.

\subsection{Statistical Indices for the performance of the models}

Statistical parameters used to estimate the generalization error so as to evaluate the performance of the models developed for the prediction. In the present work, RMSE (Root Mean Square Error), MSE (Mean Square Error) and the $\mathrm{R}^{2}$ value (Correlation Coefficient) were used as presented below:

MSE $=\frac{1}{P} \sum_{p=1}^{p}\left(d_{p}-O_{p}\right)^{2}$

$R^{2}=1-\frac{\sum_{p=1}^{p}\left(d_{p}-O_{p}\right)^{2}}{\sum_{p=1}^{p}\left(O_{p}\right)^{2}}$

$\mathrm{RMSE}=\sqrt{\frac{1}{P} \sum_{p=1}^{p}\left(d_{p}-O_{p}\right)^{2}}$

where $d_{p}$ and $o_{p}$ are the desired and calculated outputs respectively. The value of MSE and RMSE close to zero and the $R^{2}$ values close to one revealed the extent of reliability of the models $[42,43]$. Soft-computing Model's evaluation was explained based on ranges of RMSE by [44] and is given in Table 1. 
Table 1 Ranges of RMSE to analyse models performance

\begin{tabular}{ll}
\hline Ranges of RMSE & Performance \\
\hline$<0.009$ & Excellent prediction accuracy \\
$0.009<$ RMSE $<0.09$ & Good prediction accuracy \\
$0.09<$ RMSE $<0.5$ & Reasonable prediction \\
$>0.5$ & Inaccurate prediction \\
\hline
\end{tabular}

Source [44]

\subsection{Diffusion coefficient determination}

Fick's second law is applied to sweet potatoes starch drying experimental data in order to determine the effective diffusion coefficient at different drying temperatures.

$\frac{\partial c}{\partial t}=\frac{\partial}{\partial x} D \frac{\partial c}{\partial x}$

Equation 15 is solved analytically as reported by [18] and Eq. 16 is obtained:

$M R=\frac{8}{\pi^{2}} \sum_{n=0}^{\infty} \frac{1}{(2 n+1)^{2}} \exp \left[-\frac{(2 n+1)^{2} \pi^{2} D_{\text {eff }}}{4 L^{2}} t\right.$

Based on various assumptions, first term of Eq. 16 is considered and becomes:

$\ln (M R)=\frac{8}{\pi^{2}} \exp \left[-\pi^{2} \frac{D_{e f f}}{4 L^{2}} t\right]$

The effective diffusion coefficient is calculated according to Eq. 17 by plotting $\ln (M R)$ against the drying time.

\section{Results and discussion}

\subsection{Proximate composition result}

Figure 3 revealed proximate composition of dried starch at varying temperature. The ranges of the starch crude protein, fibre, ash amylose, at different temperatures, are 1.35-1.45\%, $0.3-0.39 \%, 0.21-0.28 \%$ respectively. It was observed from Fig. 3 that as the temperature increased, the crude protein content of the dried starch increased; this might be as a results of reduction in the starch moisture content as the temperature increases. It was also observed that as the drying temperature increased the crude protein and fibre increased. This occurrence might be as the result of evaporation of moisture from the starch due to increased temperature gradient. In contrast, the starch ash content decreases as drying temperature increased as shown in Fig. 3. However, amylose content profile of the starch does not follow

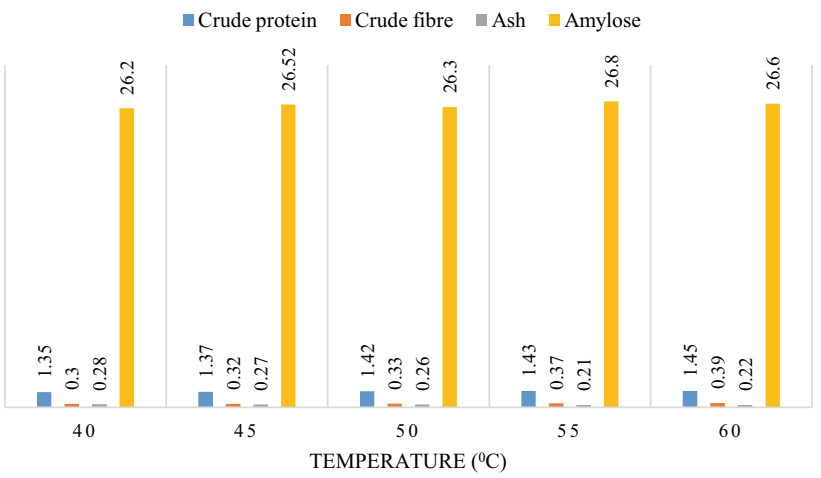

Fig. 3 Proximate composition result

the pattern. The highest and lowest amylose content was obtained at $55^{\circ} \mathrm{C}$ and $40^{\circ} \mathrm{C}$. Similar result was obtained by [45] on the influence of drying techniques on the chemical and functional properties of different potato varieties.

\section{Summary of experimental data statistics}

Table 2 shows statistics of the starch drying experimental data: drying time $\left(X_{1}\right)$, drying temperature $\left(X_{2}\right)$, ambient temperature $\left(X_{3}\right)$, relative humidity $\left(X_{4}\right)$ and moisture ratio $\left(X_{5}\right)$. The statistics comprises mean, standard error, minimum, maximum, median, standard deviation, kurtosis, skewness and sum for each variable. From Table 2, skewness for $X_{1}, X_{2}, X_{4}$ and $X_{5}$ lies between 0.16-0.72. Skewness revealed the evenness of the data dispersion; perfectly symmetric distribution that is regular involves normal skewness. However, positively skewed dispersion grouped to the left, with the tail extending to the right, while negatively skewed dispersion grouped to the right, with the tail extending to the left [46]. Therefore, the data distribution curve for $X_{1}, X_{2}, X_{4}$ and $X_{5}$ variables is relatively symmetric but the distribution data was not perfect and normal. The distribution was highly negatively skewed for $X_{3}$ with skewness less than negative one $(<-1)$. This implies that the data distribution for $X_{3}$ has scores bunched to the right, with the tail spreading to the left. The distribution curves for $X_{1}, X_{2}, X_{3} X_{4}$ and $X_{5}$ are platykurtic, because the kurtosis numeric values were less than 3 as indicated in Table 2. This suggests that the distribution curves for these variables are flatter and broader than a normal curve.

\subsection{Parametric analysis}

\subsubsection{Parameter selection for one input}

For parametric selection of starch drying investigation, four (4) exhaustive search ANFIS models were developed to explore the significance of each input factor on the 
Table 2 Descriptive statistics of input and output variables for the starch drying
Table 3 Exhaustive search regression errors (RMSE) for one-input combination

\begin{tabular}{lllllc}
\hline Statistical index & $\begin{array}{l}\text { Drying } \\
\text { tempera- } \\
\text { ture }\end{array}$ & Drying time & $\begin{array}{l}\text { Ambient } \\
\text { temperature }\end{array}$ & Relative humidity & Moisture ratio \\
\hline Mean & 48.94 & 198.63 & 75.69 & 28.43 & 0.24 \\
Standard error & 0.7 & 17.99 & 0.32 & 0.072 & 0.003 \\
Median & 50 & 150 & 76 & 28.5 & 0.243948 \\
Mode & 40 & 20 & 80 & 27.7 & 0.237879 \\
Standard Deviation & 6.83 & 175.41 & 3.15 & 0.7 & 0.032 \\
Sample Variance & 46.75 & $30,771.51$ & 9.99 & 0.497 & 0.001 \\
Kurtosis & -1.201 & 0.45 & -0.91 & -0.45 & -1.109 \\
Skewness & 0.16 & 0.72 & -0.28 & 0.036 & 0.26 \\
Range & 20 & 690 & 12 & 3.1 & 0.117839 \\
Minimum & 40 & 0 & 70 & 26.9 & 0.191664 \\
Maximum & 60 & 690 & 82 & 30 & 0.309503 \\
Sum & 4650 & 18,870 & 7191 & 2701.2 & 23.393459 \\
\hline
\end{tabular}

\begin{tabular}{lllllll}
\hline ANFIS model & Input & RMSE (train) & RMSE (test) & MSE (train) & MSE (test) & Rank \\
\hline Model1 & X1 & 0.029 & 0.0292 & 0.000841 & 0.00085264 & 2 \\
Model2 & X2 & 0.0126 & 0.013 & 0.00015876 & 0.000169 & 1 \\
Model3 & X3 & 0.0322 & 0.0322 & 0.00103684 & 0.00103684 & 3 \\
Model4 & X4 & 0.0326 & 0.0326 & 0.00106276 & 0.00106276 & 4 \\
\hline
\end{tabular}

$X 1$ temperature, $X 2$ drying time, $X 3$ ambient temperature, $X 4$ relative humidity

Table 4 Exhaustive search regression errors (RMSE) for two-input combinations
Table 5 Exhaustive search regression errors (RMSE) for three-input combinations

\begin{tabular}{lllllll}
\hline ANFIS model & Input & RMSE (train) & RMSE (test) & MSE (train) & MSE (test) & Rank \\
\hline Model1 & XIX2 & 0.009 & 0.0084 & 0.000081 & 0.00007056 & 1 \\
Model2 & XIX3 & 0.0176 & 0.0228 & 0.00030976 & 0.00051984 & 4 \\
Model3 & X1X4 & 0.0284 & 0.0364 & 0.00080656 & 0.00132496 & 6 \\
Model4 & X2X3 & 0.0098 & 0.0115 & 0.00009604 & 0.00013225 & 3 \\
Model5 & X2X4 & 0.0094 & 0.0104 & 0.00008836 & 0.00010816 & 2 \\
Model6 & X3X4 & 0.0245 & 0.0286 & 0.00060025 & 0.00081796 & 5 \\
\hline
\end{tabular}

$X 1$ temperature, $X 2$ drying time, $X 3$ ambient temperature, $X 4$ relative humidity

\begin{tabular}{lllllll}
\hline ANFIS model & Input & RMSE (train) & RMSE (test) & MSE (train) & MSE (test) & Rank \\
\hline Model1 & X1 X2 X3 & 0.0029 & 0.0062 & 0.00000841 & 0.00003844 & 1 \\
Model2 & X1 X2 X4 & 0.0031 & 0.0133 & 0.00000961 & 0.00017689 & 2 \\
Model3 & X1 X3 X4 & 0.0083 & 0.0379 & 0.00006889 & 0.00143641 & 4 \\
Model4 & X2 X3 X4 & 0.0037 & 0.0063 & 0.00001369 & 0.00003969 & 3 \\
\hline
\end{tabular}

$X 1$ temperature, $X 2$ drying time, $X 3$ ambient temperature, $X 4$ relative humidity process. The importance of input variable is ranked/or justified based on the statistical evaluation of the developed models. Tables 3,4 and 5 show the results of RMSE and MSE for both the training and testing error of the model. The ranking of the models as shown in Tables 3,4 and 5 is also based on the regression error of the models. Table 3 reveal that drying time $X_{2}$ is the most relevant variable as highlighted, while ambient temperature $\left(X_{4}\right)$ is the least influential variable as depicted in Table 3. Fundamentally, drying time is greatly affecting drying process as endorsed by previous studies [47]. The ranking of importance of the starch drying independent variables is as follows drying 
time $>$ drying temperature $>$ relative humidity $>$ ambient temperature, as shown, in Table 3. It was observed that the error difference between the most and least variables is significant. However, there is no much difference between the second to the least and the least variable as shown in Table 3. It was observed from the results that the training and testing regression errors are comparable; therefore, it is an indication that exhaustive search fitting lacks overfitting. Thus, this implies that selection of more than one variable can be further explored.

\subsubsection{Parameter selection for two and three-input combinations}

To investigate the optimal combination of two (2) parameters, six (6) possible exhaustive search NF models were developed and statistically analysed. Table 4 revealed the ranking of two-input combination influence on the starch drying. Combination of drying time and temperature is the most significant combination, as highlighted in Table 4, due to its lowest error achieved for the set of two inputs. Model 3, in Table 4, with combination of drying temperature and ambient temperature is the least ranked model. Obviously, model 3 shows the highest regression error among other models, therefore, the combination of the variables may have significant effect on the drying process. A plethora of previous researches [12, 21, 48-50] claimed and asserted that drying temperature and time are strongly influenced drying rate of agricultural produced. Therefore, the result obtained from this study shows good synergy and combined effect of drying air temperature and time on the starch drying.

Table 5 shows the parametric selection for three input combinations involved four (4) exhaustive search NF models which were statistically analysed. The result highlighted in Table 5 indicated that the combination of drying time, drying temperature and ambient temperature gave the most significant combination owing to the lowest error obtained for the three input combination using model 1 in Table 5. This result shows that the combined effect of drying time, drying temperature and ambient temperature influence the starch drying rate. It was noticed from Table 5 that NF model 3 with a combination of drying time, ambient temperature and relative humidity gave the highest regression error and therefore this combination may have low or no effect on the drying process

\subsubsection{Comparing the input parameter selection}

The evaluation of the most significant combination of 1, 2 and 3 input variables were based on the error of the training and testing values for the grid-partitioning exhaustive search model. It is noticed that as the number of relevant variable increases, the regression error decreases as shown in Fig. 4. The statistical errors for one, two and three input variable combinations were compared and three-input variable combination gave the lowest error at training and testing level as depicted in Fig. 4. This shows that three input combination has highest effect on the drying process and the combination also enhances the prediction of moisture removal during the process. It was also observed that the error discrepancy between two and three input combination was not significant; therefore, any attempt to combine more than 3 variables resulted to model overfitting and complexity. Exhaustive search model complexity leads to inaccurate representation of the process and this causes misleading results [51].

\subsection{Results of regression tree, SVM and ANFIS models}

In the Regression Tree (RT) model, Moisture Ratio (MR) was the dependent variable and Drying Temperature (DT), Drying Time (DTi), Ambient Temperature (AT) and relative humidity $(\mathrm{RH})$ were the independent variables. Three different RT models, namely, fine, medium asnd coarse RT types, were used as shown in Table 6. The results of the RT models for MR prediction are shown in Table 6. Based on the RMSE results, all the model types with different input combinations have shown good predictions with model Fine Tree and input type 4-1 having the best output with a minimum RMSE and MSE of 0.011187 and 0.00012516 respectively, while coarse tree with input type 4-1 indicating maximum RMSE and MSE. The $\mathrm{R}^{2}$ result also showed that coarse tree with input type 4-1 had poor performance due to low value of $R^{2}$ while the other showed fair predictions. It was also observed that in all the model types, the coarse tree models at all input types show the low prediction. The RMSE and MSE values obtained from regression

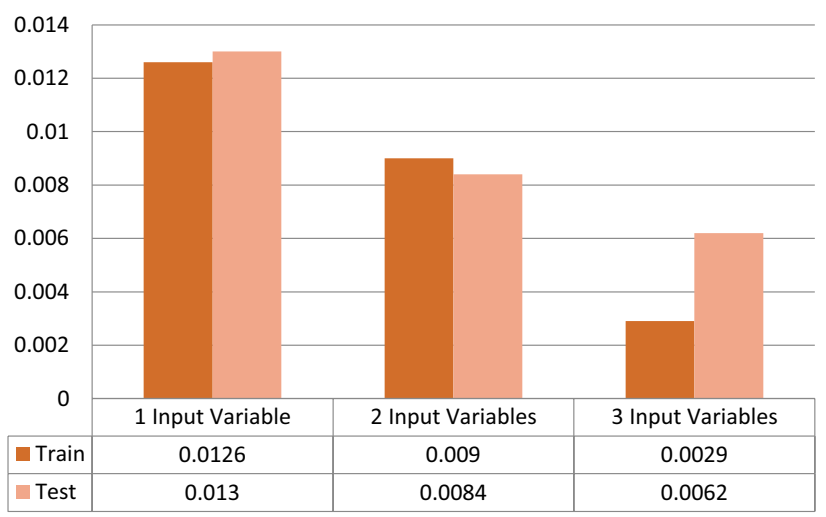

Fig. 4 RMSE for the most significant combinations of parameters with 1, 2 and 3 inputs 1 input variable =temperature, 2 input variable $=$ drying time, 3 input variable $=$ ambient temperature 
Table 6 Regression Tree (RT) model results

\begin{tabular}{|c|c|c|c|c|c|}
\hline $\begin{array}{l}\text { Input } \\
\text { type }\end{array}$ & $\begin{array}{l}\text { Model } \\
\text { type }\end{array}$ & RMSE & $\mathrm{R}^{2}$ & MSE & $\begin{array}{l}\text { Perfor- } \\
\text { mance }\end{array}$ \\
\hline RT4-1 & Fine tree & 0.011187 & 0.89 & 0.00012516 & Good \\
\hline RT 4-1 & $\begin{array}{l}\text { Medium } \\
\text { tree }\end{array}$ & 0.015911 & 0.77 & 0.00025317 & Good \\
\hline RT 4-1 & $\begin{array}{c}\text { Coarse } \\
\text { tree }\end{array}$ & 0.024274 & 0.47 & 0.00058924 & Good \\
\hline RT 3-1 & Fine tree & 0.015864 & 0.77 & 0.00025167 & Good \\
\hline RT 3-1 & $\begin{array}{l}\text { Medium } \\
\text { tree }\end{array}$ & 0.016746 & 0.75 & 0.00028044 & Good \\
\hline RT 2-1 & Fine tree & 0.013394 & 0.84 & 0.0001794 & Good \\
\hline RT 2-1 & $\begin{array}{l}\text { Medium } \\
\text { tree }\end{array}$ & 0.016583 & 0.75 & 0.000275 & Good \\
\hline RT 2-1 & $\begin{array}{c}\text { Coarse } \\
\text { tree }\end{array}$ & 0.018523 & 0.69 & 0.00034311 & Good \\
\hline
\end{tabular}

RT4- $1=4$ inputs and 1 output, RT3-1=3 inputs and 1 output, RT2-1 $=2$ inputs and 1 output

tree models were compared with previous studies on softcomputing predictive models; and comparable with existing works. On contrary, the $\mathrm{R}^{2}$ values were not similar but far from previous investigation results.

Table 7 shows SVM results with six model types (linear, quadratic, cubic, fine Gaussian, medium Gaussian and fine Gaussian SVM) for all the input types. The result for the RMSE revealed that quadratic SVM for input type 4-1 and 3-1, cubic SVM for input type 4-1, 3-1 and 2-1 and medium Gaussian SVM for input type 3-1 and 2-1 showed excellent and high prediction accuracy while the others showed good prediction accuracy in their RMSE values. The RMSE and MSE are close to zero as indicated in previous studies [22]. The $R^{2}$ values of the model showed that Cubic SVM 4-1 and 3-1 showed excellent prediction performance while quadratic SVM 4-1, 3-1 and 2-1, coarse Gaussian SVM 4-1, medium Gaussian SVM 3-1 and 2-1 and fine Gaussian SVM 2-1 showed good prediction performance in their $R^{2}$ values; whereas Linear SVM 4.1 and 2.1, medium Gaussian SVM 4.1 and fine Gaussian SVM 3.1 displayed fair performance. Fine Gaussian SVM 4.1 and linear SVM 3.1 have poor performance with Linear SVM 3.1 predicting the poorest value. This indicated that Support Vector Machine Cubic model gives outstanding predictions despite the input combinations for both the RMSE and $R^{2}$ values while linear SVM do not offer good predictions in all the input combinations

Table 8 shows subclustering ANFIS models with different radii of influence ranging from 0.6 to 1 as indicated in model types. Statistical parameters, RMSE, $\mathrm{R}^{2}$ and MSE, show the degree of agreement between estimated and measured results of the three ANFIS models (ANFIS4-1, ANFIS3-1 and ANFIS2-1) as indicated in Table 8. All the ANFIS structures, with different input types and model types are statistically and rankly excellent except ANFIS4- 1 with radius of influence 0.6 as revealed in Table 8. For 4NF-1 model types, $R^{2}$ values ranged from 0.932 to 0.99 , RMSE values from 0.0039 to 0.0131 , and MSE values from 0.00001521 to 0.00017161 . As it was also observed from Table 8, 3NF-1 model has $\mathrm{R}^{2}$ values ranging $0.9626-0.999$, RMSE values ranging from 0.0025 to 0.0088 , and MSE ranging from 0.00000625 to
Table 7 Support vector machine (SVM) model results

\begin{tabular}{llllll}
\hline Input type & Model type & RMSE & $R^{2}$ & MSE & Rank \\
\hline SVM4-1 & Linear SVM & 0.015149 & 0.79 & 0.00022949 & Good \\
SVM 4-1 & Quadratic SVM & 0.0084172 & 0.94 & $7.084 \mathrm{e}-5$ & Excellent \\
SVM 4-1 & Cubic SVM & 0.0074697 & 0.95 & $5.5722 \mathrm{e}-5$ & Excellent \\
SVM 4-1 & Fine Gaussian SVM & 0.01888 & 0.68 & 0.00035646 & Good \\
SVM4-1 & Medium Gaussian SVM & 0.013296 & 0.84 & 0.00017678 & Good \\
SVM 4-1 & Coarse Gaussian SVM & 0.011672 & 0.88 & 0.00013625 & Good \\
SVM 3-1 & Linear SVM & 0.062045 & 2.47 & 0.0038496 & Good \\
SVM 3-1 & Quadratic SVM & 0.0094437 & 0.92 & $8.9183 \mathrm{e}-05$ & Excellent \\
SVM 3-1 & Cubic SVM & 0.0070272 & 0.96 & $4.94 \mathrm{E}-05$ & Excellent \\
SVM 3-1 & Fine Gaussian SVM & 0.015206 & 0.79 & 0.00023123 & Good \\
SVM 3-1 & Medium Gaussian SVM & 0.0083571 & 0.94 & $6.9841 \mathrm{e}-5$ & Excellent \\
SVM 3-1 & Coarse Gaussian SVM & 0.012888 & 0.85 & 0.00016611 & Good \\
SVM 2-1 & Linear SVM & 0.015721 & 0.78 & 0.00024715 & Good \\
SVM 2-1 & Quadratic SVM & 0.012292 & 0.86 & 0.00015109 & Good \\
SVM 2-1 & Cubic SVM & 0.009595 & 0.92 & $9.2064 \mathrm{e}-5$ & Excellent \\
SVM 2-1 & Fine Gaussian SVM & 0.011388 & 0.88 & 0.00012968 & Good \\
SVM 2-1 & Medium Gaussian SVM & 0.0094649 & 0.92 & $8.9585 \mathrm{e}-5$ & Excellent \\
\hline
\end{tabular}

SVM4- $1=4$ inputs and 1 output, SVM3-1 = 3 inputs and 1 output, SVM2-1 = 2 inputs and 1 output 
Table 8 ANFIS sub-clustering model results

\begin{tabular}{llllll}
\hline Input type & Model type & RMSE & $\mathrm{R}^{2}$ & MSE & Performance \\
\hline ANFIS4-1 & ANFIS 0.6 & 0.0131 & 0.932 & 0.00017161 & Good \\
ANFIS 4-1 & ANFIS 0.7 & 0.0039 & 0.990 & 0.00001521 & Excellent \\
ANFIS 4-1 & ANFIS 0.8 & 0.0047 & 0.9898 & 0.00002209 & Excellent \\
ANFIS 4-1 & ANFIS 0.9 & 0.0067 & 0.9786 & 0.00004489 & Excellent \\
ANFIS 4-1 & ANFIS 1 & 0.0076 & 0.9723 & 0.00005776 & Excellent \\
ANFIS 3-1 & ANFIS 0.6 & 0.005 & 0.9878 & 0.000025 & Excellent \\
ANFIS 3-1 & ANFIS 0.7 & 0.0025 & 0.999 & 0.00000625 & Excellent \\
ANFIS 3-1 & ANFIS 0.8 & 0.0027 & 0.9966 & 0.00000729 & Excellent \\
ANFIS 3-1 & ANFIS 0.9 & 0.0047 & 0.9895 & 0.00002209 & Excellent \\
ANFIS 3-1 & ANFIS 1 & 0.0088 & 0.9626 & 0.00007744 & Excellent \\
ANFIS 2-1 & ANFIS 0.6 & 0.005 & 0.9882 & 0.000025 & Excellent \\
ANFIS 2-1 & ANFIS 0.7 & 0.0043 & 0.991 & 0.00001849 & Excellent \\
ANFIS 2-1 & ANFIS 0.8 & 0.0067 & 0.9785 & 0.00004489 & Excellent \\
ANFIS 2-1 & ANFIS 0.9 & 0.008 & 0.9692 & 0.000064 & Excellent \\
ANFIS 2-1 & ANFIS 1 & 0.0031 & 0.9358 & 0.00000961 & Excellent \\
\hline
\end{tabular}

ANFIS $4-1=4$ inputs and 1 output, ANFIS $3-1=3$ inputs and 1 output, ANFIS $2-1=2$ inputs and 1 output
0.00007744 . All the statistical values found for $3 \mathrm{NF}-1$ are generally close to acceptable range; however, 3NF-1 model with radius of neighbourhood of $0.7\left(R^{2}=0.999\right.$, $\mathrm{RMSE}=0.0025$ and MSE $=0.00000625)$ produced the best prediction among the studied ANFIS model. This indicted good agreement between estimated and measured results from $3 \mathrm{NF}-1$ model. The performance of $2 \mathrm{NF}-1$ model with influence radius $0.7\left(R^{2}=0.991, \mathrm{RMSE}=0.0043\right.$ and $\mathrm{MSE}=0.00001849$ ) is better than other radii of neigborhoods as indicated in Table 8. It was noticed from all the ANFIS models in Table 8 that the best results were obtained at radius of influence 0.7 . Comparing all model input types in Table 8, the best prediction and estimation was attained by $3 \mathrm{NF}-1$ model. The statistical results obtained from this investigation are consistent and similar to previous studies [21, 22].

\subsection{Comparison of models}

Based on MSE and $R^{2}$ values ranking, the results showed that ANFIS 3-1 is considered as the best model for the prediction of starch drying MR. It can be seen from RT, SVM and ANFIS results (Tables $6,7,8$ ) that the second to 14th best models were mostly found as ANFIS models. The excellent performance of ANFIS is due to the fact that ANFIS model is a composite model comprising linguistic representation of fuzzy logic and leaning ability of artificial neural network. Reported similar preference for ANFIS model performance among different soft-computing models in the study. However, the 15th ranked model is SVM model, while the RT models found to be the 22th30th ranked model, nevertheless, the RMSE and MSE are closed to zero as indicated in Table 6.

\subsection{Drying kinetics and diffusion coefficient determination}

Figure 5 shows the drying curve of sweet potatoes starch at different drying temperatures $\left(40-60{ }^{\circ} \mathrm{C}\right)$. It was observed from Fig. 5 that moisture equilibrium content of higher temperature was achieved at lower drying time; while longer time is required at lower temperature. It is evident that the lowest moisture contents were achieved at $55^{\circ} \mathrm{C}$ and $60^{\circ} \mathrm{C}$ respectively as shown in Fig. 5. The effect of temperature on moisture content in this study is similar to previous investigation $[19,29]$. Figure 6 showed the result of linear fitting of In (MR) against drying time (t) at different temperatures so as to obtain different slopes for estimating different effective diffusion coefficients. Correlation coefficients $\left(R^{2}\right)$ of the fittings at varying temperature are ranging from 0.928

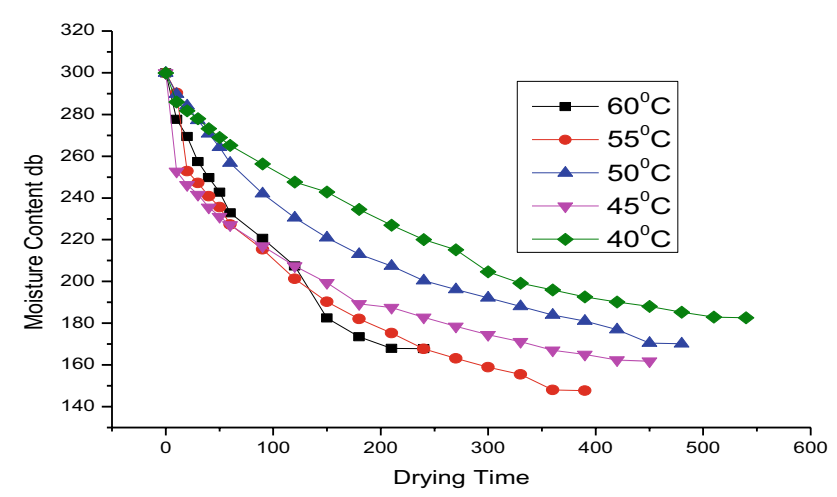

Fig. 5 Moisture content variation with time at different temperatures 


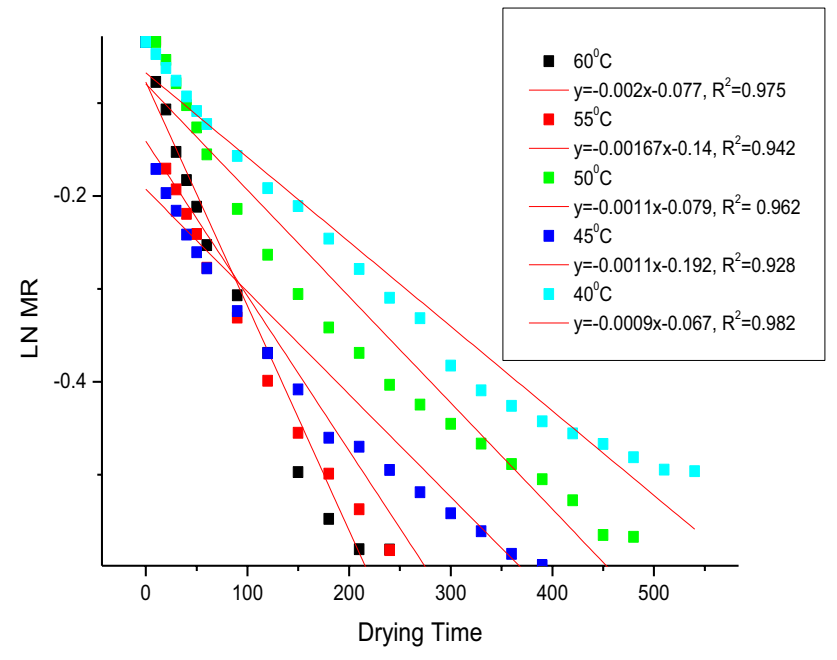

Fig. 6 Variation of InMR against drying time at different drying temperatures

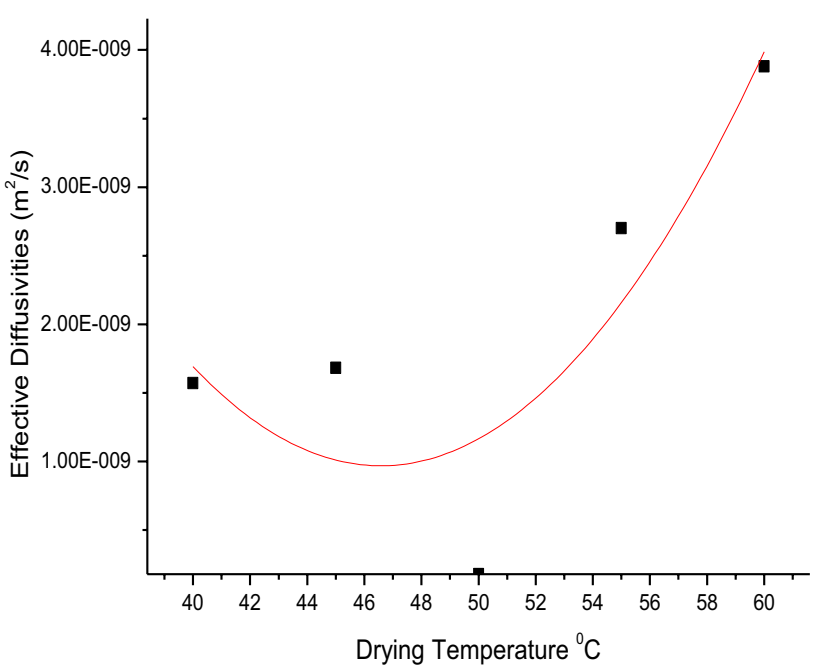

Fig. 7 Variation of diffusivity against drying temperature

to 0.982 as shown in Fig. $6 . R^{2}$ values obtained from this study are close to one, showing the reliability of the the fitting. Moreover, $R^{2}$ values for the linear fitting is comparable with the earlier investigations $[17,19]$.

Figure 7 shows the variation and distribution of moisture diffusion coefficient with drying temperatures. The effective diffusivities of sweet potatoes starch drying range from $1.78 \times 10^{-10}$ to $3.8810^{-9} \mathrm{~m}^{2} / \mathrm{s}$ at temperature $40-60^{\circ} \mathrm{C}$ as evident in Fig. 7. It was also noticed that the highest diffusivity was achieved at highest temperature; while the lowest temperature gave the lowest rate of moisture transfer from the starch. The findings from this study is related to the existing works $[5,15]$.

\section{Conclusion}

In this study, exhaustive search grid partitioning NF was used to select relevant input parameters for the prediction of starch drying performance.. Exhaustive NF parametric analysis results show that DT-DTi-AT and DT-DTi are the most influential combined variables for three and two variables combinations respectively. DTi and $\mathrm{RH}$ are also the most and least influential parameters, respectively. Among the combinations, three input combination DT-DTi-AT was selected as the optimal combination for the drying process. The 3-1NF with radius of influence 0.7 , among the models, gave the highest correlation coefficient $\left(R^{2}\right) 0.999$, lowest MSE and RSME 0.0025 and 0.00000625 respectively. The results obtained show that exhaustive search and 3-1NF models are suitable for the analysis of sweet potatoes starch drying.

This study is limited to soft-computing model development for the prediction of sweet potatoes starch. Further studies are therefore needed for the application of the neuro-fuzzy model as the dynamic equation in the fuzzybased controller design for sweet potatoes starch dryer. In addition, techno-economic analysis of sweet potatoes starch drying aspect is also recommended for further study.

\section{Compliance with ethical standards}

Conflict of interest Authors declare that there is no conflict of interest regarding the study

\section{References}

1. Adedotun H, Adebowale A-RA, Olayiwola IO, Shittu TA, Sanni LO (2015) Production and quality evaluation of noodles from sweet potato starch. J Culin Sci Technol 13(1):79-93

2. Adjei FK (2017) Comparative evaluation of the physicochemical and disintegrant properties of starch from five improved varieties of cassava in paracetamol tablet formulations. Dissertation, Knust University

3. Pilla S (2011) Starch based composites for packaging applications. Handbook of bioplastics and biocomposites engineering applications. John Wiley \& Sons, New Jersey, p 189

4. Koostanto H, Zakky M, Soebandino S (2016) Addressing the economic and healts challenges facing smallholder communities through roots and tubers in Maluku and Maluku Utara, Indonesia-results of a scoping study

5. Nwokocha LM, Aviara NA, Senan C, Williams PA (2014) A comparative study of properties of starches from Irish potato (Solanum tuberosum) and sweet potato (Ipomea batatas) grown in Nigeria. Starch-Stärke 66(7):714-723

6. Joshi KK (2017) Effect of graded doses of inorganic and organic source of nutrients on soil fertility, nutrient uptake, growth, yield and quality of Tikhur (Curcuma angustifolia 
Roxb) In Midland Inceptisols of Bastar Plateau Zone of Chhattisgarh. Dissertation, Indira Gandhi Krishi Vishwavidyalaya, Raipur (CG)

7. Tuffour E (2013) Evaluation of Starch from Ghanaian Sweet Potato Varieties as Excipients for Solid Oral Dosage Forms. Dissertation, Knust University

8. George, G.M., (2010) Virus induced gene silencing for the study of starch metabolism. Stellenbosch. Dissertation, University of Stellenbosch.

9. Monday T (2009) Industrial Sweet potato: a viable biofuel crop for Alabama. Dissertation, Auburn University

10. Aprajeeta J, Gopirajah R, Anandharamakrishnan C (2015) Shrinkage and porosity effects on heat and mass transfer during potato drying. J Food Eng 144:119-128

11. Ateeque M, Mishra RK, Chandramohan V, Talukdar P (2014) Numerical modeling of convective drying of food with spatially dependent transfer coefficient in a turbulent flow field. Int J Therm Sci 78:145-157

12. Kumar A, Deep H, Prakash O, Ekechukwu O (2017) Advancement in greenhouse drying system, solar drying technology. Springer, Singapore, pp 177-196

13. Mustayen A, Mekhilef S, Saidur R (2014) Performance study of different solar dryers: a review. Renew Sustain Energy Rev 34:463-470

14. Ray S, Raychaudhuri U, Chakraborty R (2016) An overview of encapsulation of active compounds used in food products by drying technology. Food Bioscience 13:76-83

15. Bezerra CV, da Silva LHM, Corrêa DF, Rodrigues AM (2015) A modeling study for moisture diffusivities and moisture transfer coefficients in drying of passion fruit peel. Int J Heat Mass Transf 85:750-755

16. Nadian MH, Rafiee $S$, Aghbashlo $M$, Hosseinpour $S$, Mohtasebi SS (2015) Continuous real-time monitoring and neural network modeling of apple slices color changes during hot air drying. Food Bioprod Process 94:263-274

17. Oke EO, Adeyi O, Adeyi AJ, Adekunle KF (2018) Modelling of Grewia mollis stem bark gum extraction yield using neuro-fuzzy technique. Int J Eng Res Africa Trans Tech Publ 34:70-80

18. Ariaii $P$, Tavakolipour $H$, Rezaei M, Elhami Rad AH, Bahram S (2015) Effect of methylcellulose coating enriched with Pimpinella affinis oil on the quality of silver carp fillet during refrigerator storage condition. J Food Process Preserv 39(6):1647-1655

19. Aghbashlo M, Hosseinpour S, Mujumdar AS (2015) Application of artificial neural networks (ANNs) in drying technology: a comprehensive review. Drying Technol 33:1397-1462

20. Chukwunonye CD, Nnaemeka NR, Chijioke OV, Obiora NC (2016) Thin layer drying modelling for some selected Nigerian produce: a review. Am J Food Sci Nutr Res 3:1-15

21. Prakash O, Kumar A (2014) ANFIS modelling of a natural convection greenhouse drying system for jaggery: an experimental validation. Int J Sustain Energ 33:316-335

22. Topuz A (2010) Predicting moisture content of agricultural products using artificial neural networks. Adv Eng Softw 41:464-470

23. Chegini G, Khazaei J, Ghobadian B, Goudarzi A (2008) Prediction of process and product parameters in an orange juice spray dryer using artificial neural networks. J Food Eng 84:534-543

24. Youssefi S, Emam-Djomeh Z, Mousavi S (2009) Comparison of artificial neural network (ANN) and response surface methodology (RSM) in the prediction of quality parameters of spray-dried pomegranate juice. Drying Technol 27:910-917

25. Mansor H, Noor SM, Ahmad RR, Taip F, Lutfy O (2010) Intelligent control of grain drying process using fuzzy logic controller. J Food Agric Environ 8:145-149

26. MenlikT, Özdemir MB, Kirmaci V (2010) Determination of freezedrying behaviors of apples by artificial neural network. Expert Syst Appl 37:7669-7677
27. Abakarov A, Nunez M (2012) Thermal food processing optimization: Algorithms and software. J Food Eng 163:51-59

28. dos Santos TPR, Franco CML, do Carmo EL, Jane JL, Leonel M, (2019) Effect of spray-drying and extrusion on physicochemical characteristics of sweet potato starch. J Food Sci Technol 56(1):376-383

29. Elvia D, Arifan F, Mirvanda N, Devara HR (2018) Study of process optimization of temperature and long drying of the quality and quantity of starch from sweet potato. Adv Sci Lett 24:9592-9594

30. Huang C-C, Lai P, Chen I-H, Liu Y-F, Wang C-C (2010) Effects of mucilage on the thermal and pasting properties of yam, taro, and sweet potato starches. LWT-Food Sc Technol 43:849-855

31. Li S, Ye F, Zhou Y, Lei L, Zhao G (2019) Rheological and textural insights into the blending of sweet potato and cassava starches: in hot and cooled pastes as well as in fresh and dried gels. Food Hydrocoll 89:901-911

32. Nuriyah L, Saroja G, Ghufron M, Razanata A, Rosid NF (2018) Tensile strength and elongation characteristics of bioplastics made from cilembu sweet potatoes starch with various types of plasticizers. Natural B 4:177-182

33. Wu Q, Qu H, Jia J, Kuang C, Wen Y, Yan H, Gui Z (2015) Characterization, antioxidant and antitumor activities of polysaccharides from purple sweet potato. Carbohyd Polym 132:31-40

34. AOOA, (1990) Official methods of analysis of the Association of Official Analytical Chemists

35. Chiu SL (1994) Fuzzy model identification based on cluster estimation. J Intell Fuzzy Syst 2:267-278

36. Lohany B, Reddy P, Mishra RK (2006) Airbone altimedtric lidar simulator: an educational tool, international archives of the photogrammetry, remote sensing and spatial information. Science 36(6):179-183

37. Vapnik V (2013) The nature of statistical learning theory. Springer science \& Business media, Berlin

38. Bray $M, \operatorname{Han} D$ (2004) Identification of support vector machines for runoff modelling. J Hydroinform 6:265-280

39. Mohandes MA, Halawani TO, Rehman S, Hussain AA (2004) Support vector machines for wind speed prediction. Renew Energy 29:939-947

40. Kisi and Cimen M. (2009) Evapotransporation modelling using support vector machines. Hydrol Sci J 54(5):918-928

41. Zhou H, Li W, Zhang C, Liu J (2009) Ice breakup forecast in the reach of the Yellow river: the support vector machines approach. Hydrology and Earth System Sciences Discussions 6:3175-3198

42. Oke E, Arinkoola A, Salam K (2014) Mathematical modeling of mass transfer rate during injection of $\mathrm{CO}_{2}$ into water and surfactant solution. Pet Coal 56:54-61

43. Kalogirou SA (2000) Applications of artificial neural networks for energy systems. Appl Energy 67:17-35

44. Li M, Fan L, Liu H, Guo P, Wu W (2013) A general model for estimating daily global solar radiation using air temperatures and site geographic parameters in Southwest China. J Atmos Solar Terr Phys 92:145-150

45. Kolawole F, Balogun M, Akeem S, Salaudeen L (2018) Effect of drying methods on the yield, phytochemical composition and antioxidant activities of potato (solanum tuberosum) and two sweet potato (ipomoea batatas) varieties. Carpathian J Food Sci Technol 10(2):107-119

46. Ahmed S, Zhou Z, Pang Y, Xu Y, Tong C, Bao J (2018) Genetic diversity of potato genotypes estimated by starch physicochemical properties and microsatellite markers. Food Chem 257:368-375

47. Adeyi O, Adeyi AJ, Oke EO (2018) Empirical modelling of thin layer drying characteristics of nuclear Latifolia leaves. J Niger Soc Chem Eng 33(1):17-25 
48. Kumar R, Aggarwal R, Sharma J (2015) Comparison of regression and artificial neural network models for estimation of global solar radiations. Renew Sustain Energy Rev 52:1294-1299

49. Zotarelli MF, Carciofi BAM, Laurindo JB (2015) Effect of process variables on the drying rate of Mango Pulp by retractance windo. Food Res Int 69:410-417

50. Oke EO, Araromi DO, Jimoda LA, Adetayo Adeniran J (2019) Kinetics and neuro-fuzzy soft computing modelling of river turbid water coag-flocculation using mango (Mangifera indica) kernel coagulant. Chem Eng Commun 206(2):254-267
51. Tabari H, Kisi O, Ezani A, Talaee, (2012) P.H., SVM, ANFIS, regression and climate based models for reference evapotranspiration modeling using limited climatic data in a semi-arid highland environment. J Hydrol 444:78-89

Publisher's Note Springer Nature remains neutral with regard to jurisdictional claims in published maps and institutional affiliations. 